\title{
openheart Time trends in the risk of atrial fibrillation and ischaemic stroke in patients with peripheral artery disease between 1997 and 2015
}

\author{
Sadaf Kamil (1) , ${ }^{1,2}$ Thomas Sehested, ${ }^{2}$ Kim Houlind, ${ }^{3,4}$ Jens Lassen, ${ }^{5}$ \\ Gunnar Gislason, ${ }^{1,6}$ Helena Dominguez ${ }^{2,7}$
}

\begin{abstract}
- Additional material is published online only. To view please visit the journal online (http://dx.doi.org/10.1136/ openhrt-2019-001185).
\end{abstract}

To cite: Kamil S, Sehested T, Houlind $\mathrm{K}$, et al. Time trends in the risk of atrial fibrillation and ischaemic stroke in patients with peripheral artery disease between 1997 and 2015. Open Heart 2020;7:e001185.

doi:10.1136/

openhrt-2019-001185

The abstract of the present study has been presented as a poster at ESC Congress 2019 (Paris); however, the data have never been published in any scientific journal.

Received 7 0ctober 2019 Revised 1 March 2020 Accepted 24 March 2020
Check for updates

(C) Author(s) (or their employer(s)) 2020. Re-use permitted under CC BY-NC. No commercial re-use. See rights and permissions. Published by BMJ.

For numbered affiliations see end of article.

Correspondence to

Dr Sadaf Kamil; sadafkamil88@ gmail.com

\section{ABSTRACT}

Objective The risk of atrial fibrillation (AF) and stroke in patients with peripheral artery disease (PAD) is an important issue that has not been investigated adequately. Our aim with the present study was to explore trends in the incidence of AF and stroke in patients with PAD. Methods We employed Danish nationwide registers to identify all patients with first-time diagnosis of PAD (aged $\geq 18$ years) between 1997 and 2015. Age-standardised incidence rates per 1000 person-years were calculated to estimate trends of $\mathrm{AF}$ and stroke. Risk of $\mathrm{AF}$ and stroke was estimated by 1 year cumulative incidence.

Results A total of 121.241 patients with first-time diagnosis of PAD were identified. The 1-year cumulative incidence of AF in patients with PAD were 1.97\% for year $1997-2000,2.63 \%$ for year $2001-2005,2.66 \%$ for year 2006-2010 and $2.78 \%$ for year 2011-2015, respectively. The 1-year cumulative incidence of stroke in patients with PAD were $2.71 \%, 2.71 \%, 1.95 \%$ and $1.81 \%$, for the 1997-2000, 2001-2005, 2006-2010 and 2011-2015year groups, respectively. Likewise, the age-standardised incidence rates showed increasing trends of AF during the study period, whereas trends of stroke demonstrated a decline. During study, the initiation of cholesterol-lowering agents and clopidogrel increased markedly from $7.0 \%$ to $51.3 \%$ and $0.1 \%$ to $5.9 \%$, whereas use of warfarin slightly dropped from $4.29 \%$ to $3.21 \%$.

Conclusions The incidence of $A F$ in patients with $P A D$ has significantly increased over time, whereas a marked decline has occurred in the incidence of stroke. This may suggest that the secondary prevention strategies aimed at reducing risk of stroke are broadly effective.

\section{INTRODUCTION}

Peripheral artery disease (PAD) is associated with increased risk of other cardiovascular disease and is considered as a clinical manifestation of systemic atherosclerosis. ${ }^{1}$ More than 200 million individuals worldwide are estimated to have PAD, and the prevalence continues to rise with increasing age. ${ }^{2}$ Atrial fibrillation $(\mathrm{AF})$ is the most prevalent cardiac arrhythmia that is associated with increased risk of stroke. ${ }^{3}$ Recent observational studies

\section{Key questions}

What is already known about this subject?

- Peripheral artery disease (PAD) is associated with increased risk of cardiovascular disease and is considered as a clinical manifestation of systemic atherosclerosis.

What does this study add?

- In this retrospective register-based cohort study, the age-standardised incidence of atrial fibrillation (AF) in patients with PAD has significantly increased over time, whereas a marked decline has occurred in the incidence of stroke. Global ageing, increased focus on diagnosis and improved cardiovascular pharmacotherapy may explain the increasing incidence of $\mathrm{AF}$ and declining risk of stroke.

How might this impact on clinical practice?

- The results from the present study highlight the enormous impact this may have on future healthcare costs and expenses. Further consideration on impact of our findings on the clinical management, as well as additional studies on the mechanistic links between PAD, $A F$ and stroke, are required, which may aid in reducing the cardiac morbidity burden and the mortality rate in PAD population.

have reported a high prevalence of $\mathrm{PAD}$ $(12.2 \%-16.8 \%)$ in patients with $\mathrm{AF}^{4}$ Indeed, $\mathrm{PAD}$ and AF share many common cardiovascular risk factors, including hypertension, hypercholesterolaemia and diabetes mellitus (DM). ${ }^{4-6}$ It is also well known that coexistence of AF and stable vascular disease (including PAD) substantially increases the risk of future cardiovascular events; hence, PAD is included as a prognostic factor in congestive heart failure, hypertension, age $\geq 75$ years, DM, prior stroke, vascular disease, age 65-75 years and sex category (CHA2DS2VASc) risk score. ${ }^{7}$ An early detection and timely treatment of clinical risk factors in patients with $\mathrm{PAD}$ and $\mathrm{AF}$ may therefore 
contribute in reducing the risk of cardiovascular adverse outcomes. Furthermore, it is of paramount importance to gain insight into the $\mathrm{PAD}$ and $\mathrm{AF}$ population profile and plan future interventional studies to improve the public health burden related to increased risk of stroke, morbidity and death in these patients. To the best of our knowledge, data on temporal trends of $\mathrm{AF}$ and stroke incidence in patients with PAD are sparse; therefore, we intended to estimate the age-standardised annual incidence of $\mathrm{AF}$ and ischaemic stroke in all Danish citizens diagnosed with PAD over a 20-year period.

\section{METHODS \\ Data sources}

All Danish residents are allocated a permanent and unique civil registration number at birth or immigration that enables unambiguous cross-linkage of data across several national administrative and healthcare registers. ${ }^{8-10}$ These registers are nationwide and offer a unique possibility of conducting large-scale epidemiological studies of several endpoints with a minimum loss to follow-up. In the present study, the Danish National Patient Registry was used to retrieve information on comorbidities, prior to the PAD diagnosis date. ${ }^{9}$ This register holds information on all inpatient and outpatient treatments (recorded as International Classification of Diseases (ICD) codes) since 1978. Data on related pharmacotherapy were gained from the Danish Register of Medicinal Product Statistics (National Prescription Register) that holds information on all medicine prescribed and dispensed since 1995 according to The International Anatomical Therapeutic Chemical (ATC) classification system. ${ }^{9} 10$ These Danish registers have previously been shown to be complete and accurate with a number of well-validated diagnoses codes. ${ }^{9-11}$

\section{Study population}

The study cohort included all Danes aged $\geq 18$ years, with a first-time diagnosis of PAD (inpatient and outpatient) over a period of 19 years spanning from 1 January 1997 to 31 December 2015. Patients with a previous diagnosis of PAD, AF and stroke were excluded at baseline. Moreover, the study subjects were censored on death, migration and at the end of study period (31 December 2015). Definition of PAD is based on European Society of Cardiology (ESC) Guidelines on the Diagnosis and Treatment of Peripheral Arterial Diseases (2017) and comprises all arterial diseases (ICD-10 diagnoses codes: I70, I702, I708, I709, I738, I739, I742, I743, I744, I748 and I749) except the atherosclerotic disease of the coronary arteries, aorta and intracranial arteries.

\section{Pharmacotherapy and comorbidity}

Baseline pharmacotherapy was defined by dispensed prescriptions 180 days prior to the PAD diagnosis date. Comorbidities were established based on diagnostic codes recorded in the National Patient Registry, within 10 years of diagnosis of PAD. Hypertension was identified by hospital diagnoses for hypertension (ICD-10 codes I110-I115) or if patient was treated with at least two of the following antihypertensive agents inside a 90-day period after receiving the diagnosis: alpha-adrenergic blockers, non-loop diuretics, vasodilators, beta-blockers, calcium channel blockers and renin-angiotensin system inhibitors. ${ }^{12}$ The respective ICD and ATC codes for all examined comorbidities and pharmacotherapy are presented in online supplementary table.

\section{Outcomes}

$\mathrm{AF}$ and ischaemic stroke were outcomes of interest and defined by the ICD-10 diagnoses codes, that is, I48, I63 and I64. Haemorrhagic stroke (I61) was not considered for this study. Patients with embolic stroke of undetermined source are predominantly coded as I64 in the Danish registers.

\section{Statistical analysis}

All statistical analyses were performed with SAS statistical software V.9.4, R statistics (R Core Team, 2016), and Joinpoint regression program (4.2.0.1 May 2015, National Cancer Institute, https://surveillance.cancer.gov/joinpoint/).

Baseline characteristics for cohort participants were presented as medians, frequencies and percentages. $\chi^{2}$ test was used to test the difference between categorical variables and t-test or the Kruskal-Wallis test for differences between continuous variables. The level of statistical significance was set as $\mathrm{p}<0.05$. Risk of $\mathrm{AF}$ and stroke in 1 year of follow-up were presented as cumulative incidence curves with 95\% CI using the Aalen-Johansen method. Risk-time (used to calculate cumulative incidence) was constituted of time since PAD diagnosis date (index) until an outcome of either AF or stroke occurred. Furthermore, age-adjusted time trends of PAD were estimated as incidence rates per 1.000 person-years (IR). Age was categorised in following age groups (years): 18-49, 50-59, 60-69 and $>70$.

\section{Ethics}

Data were made available in a way that individuals could not be identified. Approval from an ethics committee is not required for retrospective register-based studies in Denmark.

The study was conducted and reported in accordance with the recommendation of Strengthening the Reporting of Observational Studies in Epidemiology. ${ }^{13}$

\section{Patient and public involvement}

Patients or the public were not involved in the design, or conduct, or reporting, or dissemination of our research.

\section{RESULTS}

After exclusion of patients with prevalent AF, PAD or stroke at baseline, a total of 121.241 patients had a firsttime diagnosis of PAD between 1 January 1997 and 31 December 2015. Mean age was 68.89 (IQR 61-77 years), 
All patients diagnosed $1^{\text {st }}$ time with PAD aged $\geq 18$ years

between 01 Jan 1997 - 31 Dec 2015 $n=152.849$

Excluded at baseline:

Prevalent atrial fibrillation $n=17.273$

Prevalent stroke $n=14.365$

Total study population $\mathrm{n}=121.211$

Figure 1 Flow chart of selection of the study population. PAD, peripheral artery disease.

and $53.05 \%$ of the identified patients were male. A flow chart of the study population is illustrated in figure 1 and baseline characteristics stratified by four year-groups with 5-year intervals among patients with PAD are presented in table 1.

During study period, the initiation of cholesterollowering agents, acetylsalicylic acid, clopidogrel and new oral anticoagulants increased markedly from $7.0 \%$ to $51.3 \%, 30.1 \%$ to $47.7 \%, 0.1 \%$ to $5.9 \%$ and $0.0 \%$ to $0.7 \%$, respectively, whereas use of vitamin $\mathrm{K}$ antagonist declined from $4.3 \%$ to $3.2 \%$ (table 1 ).

A total of $15.476(12.77 \%)$ patients with PAD developed AF, and $14.671(12.10 \%)$ patients with PAD were diagnosed with stroke the following years. The agestandardised incidence rates showed increasing trends of AF from IR 24.44 (1997) to IR 33.20 (2015) per 1.000 person-years during the study period, while trends of stroke demonstrated a decline from IR 34.51 (1997) to IR 18.58 (2015) per 1.000 person-years (figure 2). The estimated annual per cent change for the entire time period (1997-2015) increased by $1.7 \%$ (95\% CI 0.8 to 2.7, $\mathrm{p}<0.0001)$ for AF and decreased by $3.4 \%$ (95\% CI 2.4

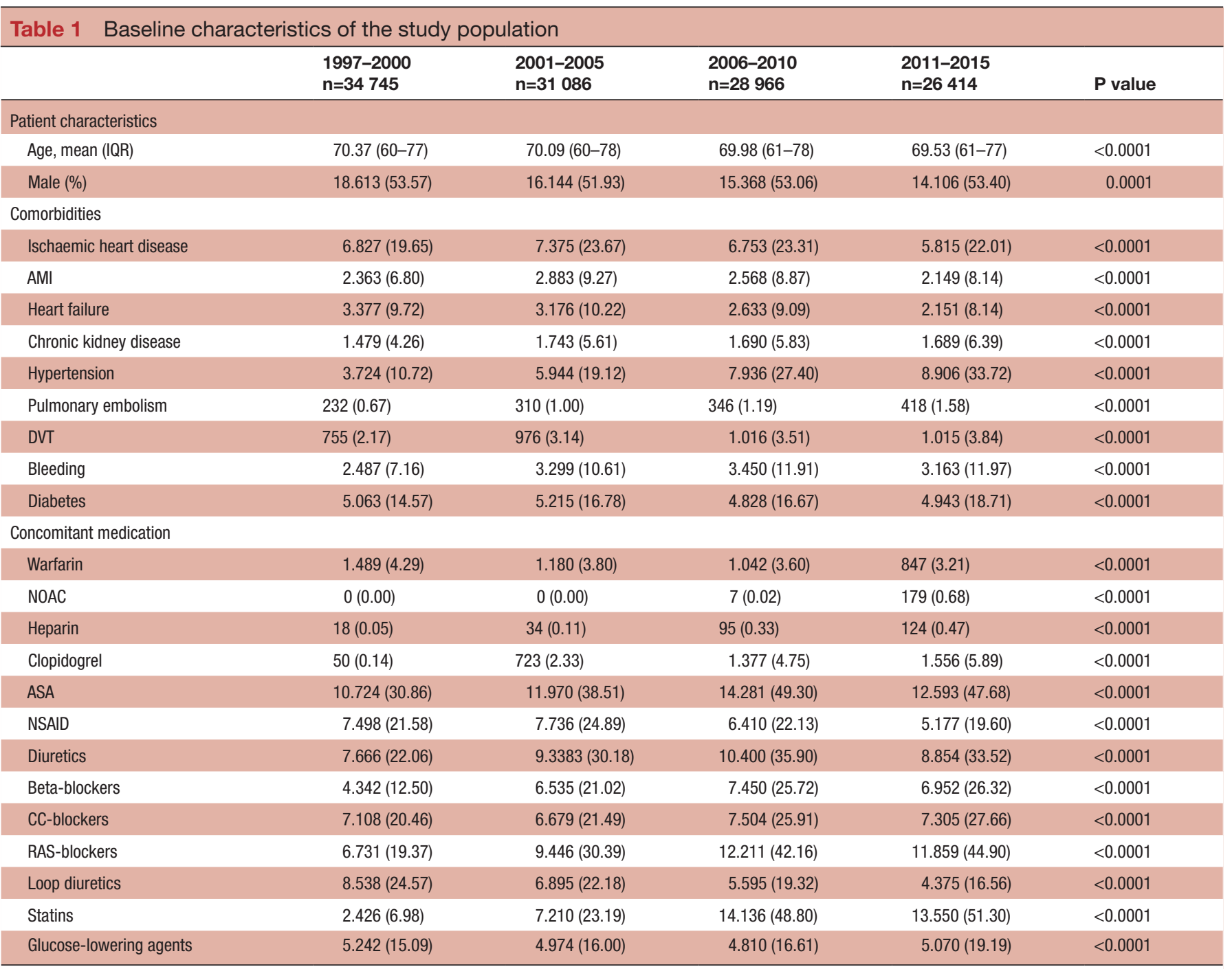

Continuous variables are presented as means (with SD values) and discrete variables as percentages (\%).

AMI, acute myocardial infarction; ASA, acetylsalicylic acid; CC-blockers, calcium channel blockers; DVT, deep venous thrombosis; NOACs, new oral anticoagulants; NSAID, non-steroidal anti-inflammatory drugs; RAS-Blockers, renin-angiotensin converting system blockers. 
Incidence of Atrial Fibrillation

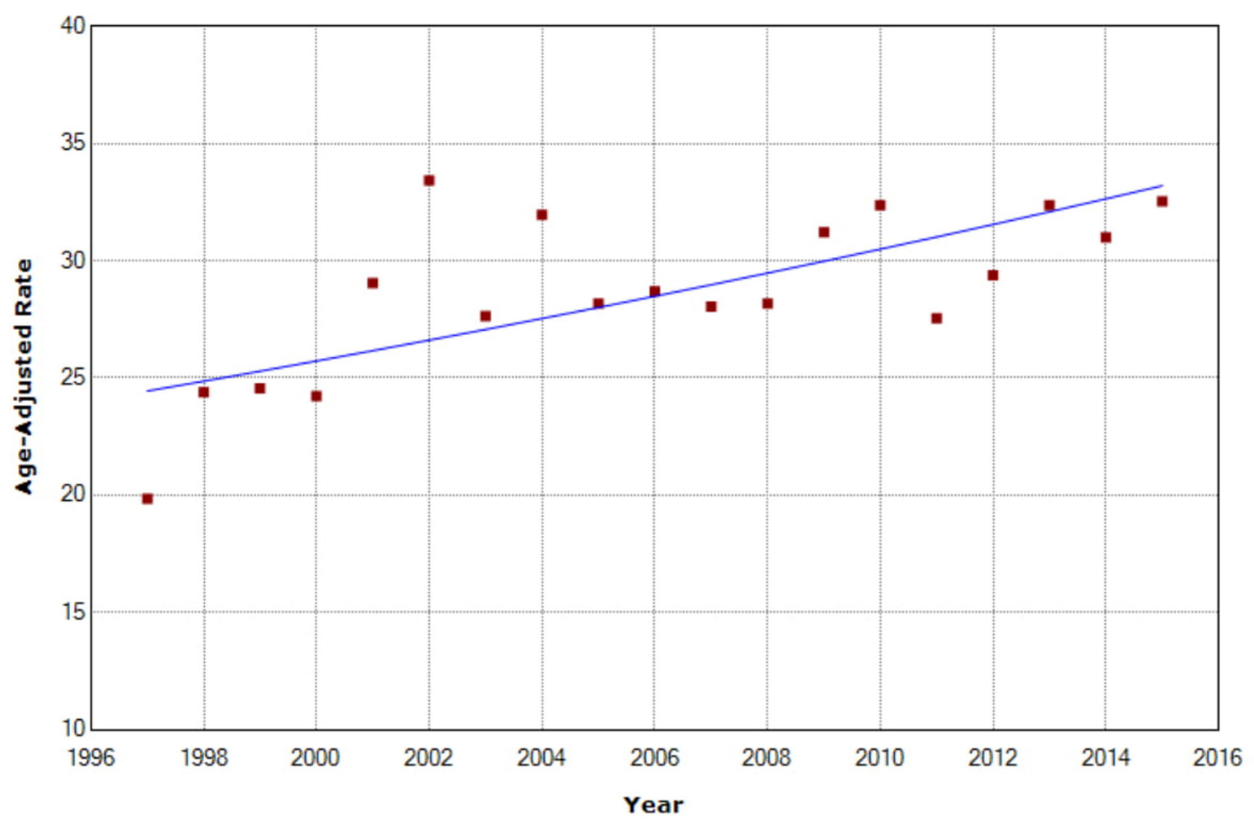

Incidence of Stroke

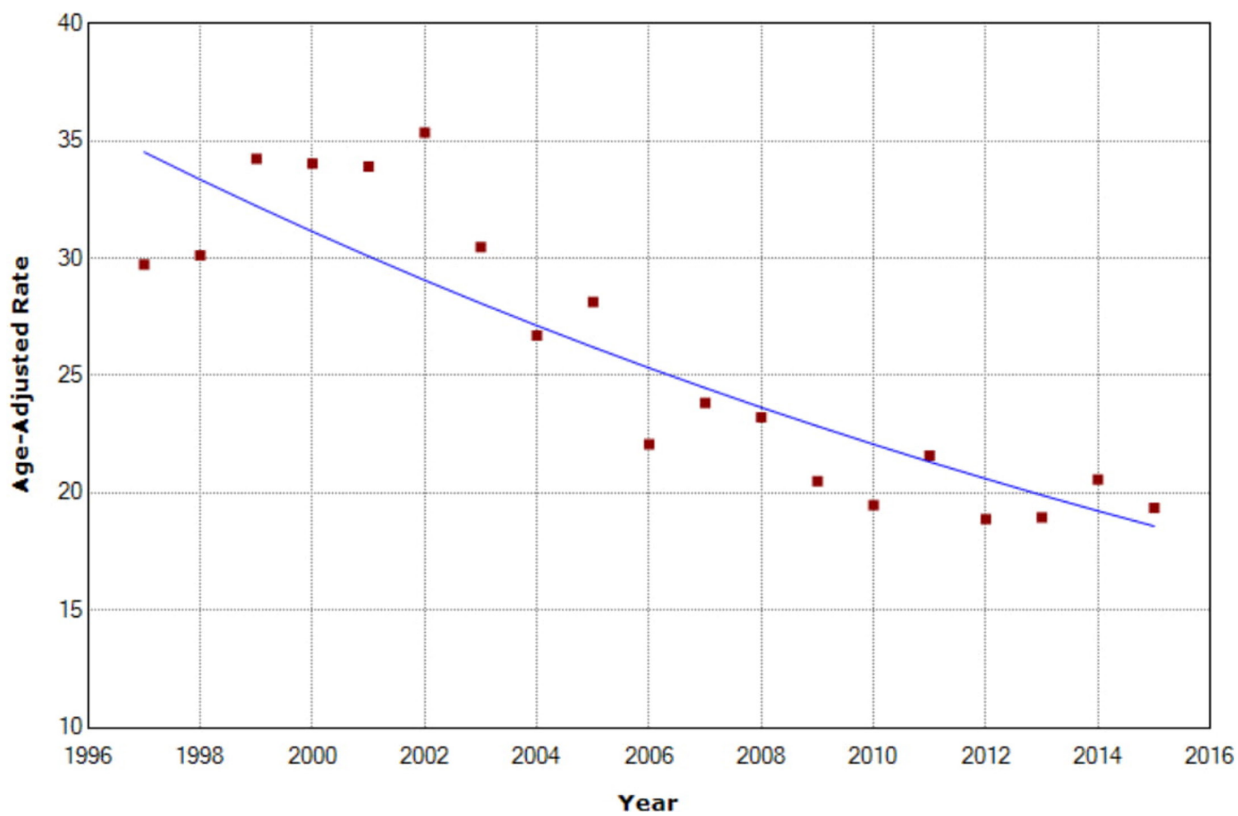

Figure 2 Age-standardised incidence rates per 1.000 person-years of AF and stroke in patients with PAD (y-axis) from 1997 to 2015 (x-axis). AF, atrial fibrillation; PAD, peripheral artery disease.

to $4.3, \mathrm{p}<0.0001)$ for stroke. All age-standardised trends were statistically significant $(\mathrm{p}<0.05)$.

In addition, the 1-year cumulative incidence of $\mathrm{AF}$ in patients with PAD were estimated to be $1.97 \%$ for year $1997-2000,2.63 \%$ for year 2001-2005, 2.66\% for year 2006-2010 and $2.78 \%$ for year 2011-2015, respectively. The corresponding 1-year cumulative incidence of stroke in patients with PAD were $2.71 \%, 2.71 \%, 1.95 \%$ and $1.81 \%$, for the 1997-2000, 2001-2005, 2006-2010 and 2011-2015 year-groups, respectively (figure 3).
Furthermore, an additional subcategory analyses to examine the specific trends for PAD diagnoses codes (I70, I73, and I74) is included as supplementary data (online supplementary file 2).

\section{DISCUSSION}

In this Danish nationwide register-based cohort study including patients with first-time diagnosis of PAD, we examined temporal trends on risk of $\mathrm{AF}$ and stroke 

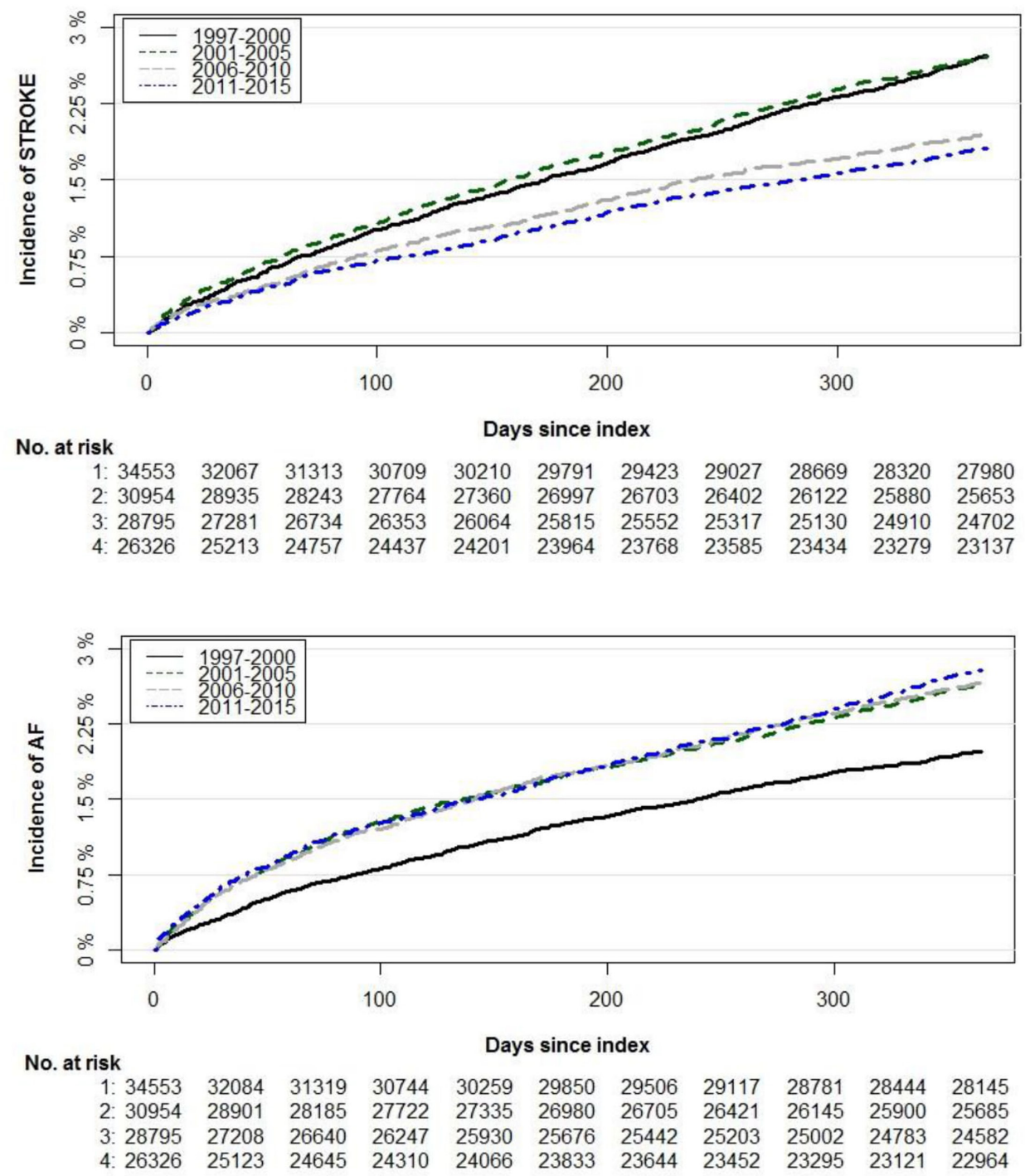

Figure 3 Cumulative incidence curves and number of patients at risk within 1 year from first-time diagnosis of PAD to development of AF or stroke. At-risk table represents number of patients at risk of an event at given time (ie, each month for all four time periods). AF, atrial fibrillation; PAD, peripheral artery disease.

spanning over 20 years. The main finding is a significantly increased age-adjusted incidence of AF and a significant decline in the incidence of stroke along the study period. Moreover, the corresponding 1-year cumulative incidence of $\mathrm{AF}$ in patients with PAD showed an increasing trend, whereas the 1-year cumulative incidence of stroke in patients with PAD demonstrated a decline.

PAD is considered as a serious atherosclerosis-related disease that can lead to frequent hospitalisations, higher health economic cost, a poor quality of life and an increased mortality rate. ${ }^{14-16}$ Although PAD definition is that of atherosclerosis of the arteries outside the heart, the term is most commonly used to describe occlusive arterial disease of the lower extremities. ${ }^{17}$

Similar to other atherosclerotic diseases, PAD is associated with the presence of traditional cardiovascular risk factors, including DM, hypertension, dyslipidaemia, obesity and tobacco consumption. ${ }^{5}{ }^{17}$ Moreover, along with endothelial dysfunction and hypercoagulable state, studies have linked PAD with a heightened low-grade inflammation that may contribute to increased risk of cardiovascular events. ${ }^{18-20}$ Indeed, patients with PAD are at remarkably high risk of cardiovascular and cerebrovascular diseases, which are concomitantly present in more than half of the patients with PAD. ${ }^{21}$

It is commonly known that AF is strongly correlated to atherosclerotic vascular disease. ${ }^{22}$ As PAD is one of the cardinal signs representing the presence of systemic atherosclerosis, AF and PAD often coexist. ${ }^{323}$ Furthermore, restricted physical activity due to intermittent claudication may aid the development of adverse conditions, such as insulin resistance, metabolic syndrome, obesity and severe depression, which confer an increased cardiovascular risk. Recent observational studies have reported a high prevalence of PAD (12.2\%-16.8\%) in patients with $\mathrm{AF}$ and an increased risk of incident $\mathrm{AF}(29 \%)$ in patients with PAD. ${ }^{4}$ Although the complex mechanism linking AF and PAD is not fully elucidated, mounting evidence has 
suggested an overlap of multiple common risk factors, increased levels of inflammation and prothrombic state, which leads to the susceptibility for a bidirectional relationship between the two conditions. This association between $\mathrm{AF}$ and PAD elicits an increase in all major adverse cardiovascular outcomes, including stroke and thromboembolic events, which makes the concurrent presence a much dangerous combination for patients with $\mathrm{AF}$ and PAD. ${ }^{323}$ In addition, PAD is also considered as an independent predictor of ischaemic stroke, which is the main reason that it is currently included in the clinical score (CHA2DS2-VASc score) of baseline assessment for newly diagnosed patients with $\mathrm{AF}^{724}$ The overlap of the common risk factors among $\mathrm{PAD}, \mathrm{AF}$ and stroke reflect shared pathophysiological mechanisms and a multiplestage disease process, where presence of risk factors promotes development of atherosclerosis, diagnosis of $\mathrm{PAD}$, incident $\mathrm{AF}$ and ultimately thromboembolic events including stroke.

Despite the well-known association and evidence on the prognostic implication of $\mathrm{AF}$ in $\mathrm{PAD}$, there are only a few studies, if any, that have evaluated the trends of AF and stroke in patients with PAD during the past two decades. We therefore used the Danish national health and administrative registers to examine this important topic. The results from our study demonstrate an increasing incidence of AF in patients with PAD across years. This trend is supported by other studies suggesting a growing prevalence and incidence of AF linked to an early and frequent diagnosis and the shifting demographics of the general populations. ${ }^{25-30}$ For example, recent estimates from the Framingham cohort reported a 3-4 fold increase in ageadjusted incidence of AF between 1958 and 1967 and 1998 and 2007, along with temporal changes in AF-associated risk factors. ${ }^{31}$ Indeed, as people age, they are more likely to develop chronic medical conditions, including $\mathrm{PAD}$ and $\mathrm{AF}$. In addition to ageing population and improved survival from other cardiovascular conditions, the increased awareness and initiatives to improve the detection of $\mathrm{AF}$ is also considered as a major contributing factor in greater incidence of diagnosed $\mathrm{AF}^{26}$ Moreover, $\mathrm{PAD}$ and $\mathrm{AF}$ share several risk factors, for example, increased levels of inflammation, thrombosis and coagulation, which may, in part, explain the increased incidence of AF in PAD patients. ${ }^{18-20}$ Nevertheless, further research is needed to clarify the underlying mechanisms.

Furthermore, the present study demonstrates a consistent decline in the incidence of stroke during the study period of 20 years. This trend is in line with the studies demonstrating the similar lower incidence of stroke in general population. ${ }^{27}$ Patients with PAD are at increased risk of stroke due to more advanced atherosclerosis, increased prevalence of comorbidities, enhanced platelet aggregability and a reduced fibrinolytic potential. ${ }^{32}$ Preventing future ischaemic events is therefore the main objective of the PAD treatment. The observed decrease in age-standardised rates and cumulative incidence of stroke in the present study may suggest an increased and effective use of anticoagulants and antiplatelet drugs and a better control of modifiable cardiovascular risk factors, particularly hypertension, diabetes, dyslipidaemia and smoking. ${ }^{33}{ }^{34}$ Interestingly, during the course of our study (ie, between 1997 and 2015), the use of cholesterol-lowering agents, antihypertensives and clopidogrel increased markedly, which supports the notion of improvement in risk factor modification strategies in high-risk population (table 1). Hypertension is the most prevalent risk factor for stroke, and it is estimated that the risk of stroke could be significantly reduce through an optimal management of hypertension..$^{35}$ Consequently, the treatment guidelines have included recommendations focused on lowering blood pressure in patients with hypertension-related conditions including stroke. ${ }^{36}$ Comparable with the trends observed in the general population, we also observe an increase in use of antihypertensive treatment in patients with PAD in the present study (table 1). The similar increase has been observed in prevalence of hypertension, which has increased from $10.7 \%$ (1997-2000) to $33.7 \%$ (2011-2015). As we have defined hypertension by using diagnoses coded and/or use of at least two antihypertensive agents, this may, in part, explain the observed higher frequency. Also, this increase is in line with the existing studies showing an overall increased risk of hypertension in PAD, as well as an increase prevalence of hypertension in general population during the recent years. ${ }^{37-40}$

Taken together, besides constituting highly relevant future research questions, the results from the present study underline the importance of timely and early anticoagulation in management of patients with PAD in order to prevent risk of thromboembolic events including stroke. Of notice, PAD is often overlooked by the primary care providers as the early clinical findings are subtle and patients may not complain of the symptoms until the disease is in the advance stages. More attention should be paid to patients in whom coexistence of $\mathrm{AF}$ and $\mathrm{PAD}$ occurs, as they represent a vulnerable subgroup at a very high risk for adverse outcomes. Indeed, AF in patients with $\mathrm{PAD}$ may serve as a surrogate marker of more severe disease with subsequent poor cardiovascular (CV) outcomes in terms of survival and morbidity. Showing a substantial increase in AF incidence in patients with $\mathrm{PAD}$, the results from the present study highlights the enormous impact this may have on future healthcare costs and expenses. Nevertheless, further consideration on impact of our findings on the clinical management, as well as additional studies on the mechanistic links between PAD, $\mathrm{AF}$ and stroke, are required, which may aid in reducing the cardiac morbidity burden and the mortality rate in PAD population.

\section{Strengths and limitations}

Notable strengths of the present study include a large number of unselected patients in a real-world setting, completeness of follow-up and use of validated measures of exposure and outcome. Furthermore, the Danish 
healthcare system is government financed that guarantees free of charge and equally accessible care for all its inhabitants, reducing confounding by variables associated with social class to a minimum.

Despite these strengths, there are also several limitations that must be acknowledged. For example, the observational nature of the study only enables to establish association and does not represent cause-and-effect relationships. Moreover, the subpopulation was identified by using diagnosis from the registers; although the registration of data in Danish registers is known to be accurate, there still might be some limitations regarding the PAD diagnoses, especially as PAD is under-reported due to the often-asymptomatic nature of the disease. Finally, the Danish population is predominately of Northern European descent, and generalisability of our findings to other ethnicities should be performed with caution.

\section{CONCLUSION}

The incidence of AF in patients with PAD has significantly increased over time, whereas a marked decline has occurred in the incidence of stroke. This may suggest that the secondary prevention strategies aimed at reducing risk of stroke are broadly effective. Moreover, an increased focus on earlier and adequate diagnosis and improved treatment of cardiovascular disease allowing survival with the disease may explain the increasing incidence of AF.

\author{
Author affiliations \\ ${ }^{1}$ Department of Cardiology, Gentofte Hospital, Hellerup, Denmark \\ ${ }^{2}$ Department of Cardiology, Bispebjerg Frederiksberg Hospital, Copenhagen, \\ Denmark \\ ${ }^{3}$ Department of Vascular Surgery, Kolding Hospital, Kolding, Denmark \\ ${ }^{4}$ Department of Regional Health Research, University of Southern Denmark, \\ Odense, Denmark \\ ${ }^{5}$ Department of Cardiology, Odense University Hospital, Odense, Denmark \\ ${ }^{6}$ The Danish Heart Foundation, Copenhagen, Denmark \\ ${ }^{7}$ Department of Biomedicine, Copenhagen University, Copenhagen, Denmark
}

Acknowledgements The study sponsors had no influence on data collection, interpretation of data, nor the final decision to submit the manuscript for publication.

Contributors SK, TS, HD and GG designed the study. SK performed the statistical analyses and wrote the initial draft of the paper. All authors contributed equally to interpretation of data, revised the paper for important intellectual content and approved the final version of the paper.

Funding The authors have not declared a specific grant for this research from any funding agency in the public, commercial or not-for-profit sectors.

Competing interests None declared.

Patient consent for publication Not required.

Ethics approval The Danish Data Protection Agency approved the present study (ref. 2007-58-0015, int. ref: GEH-2014-018).

Provenance and peer review Not commissioned; externally peer reviewed.

Data availability statement Data may be obtained from a third party and are not publicly available. Data may be obtained from a third party on reasonable request and are not publicly available.

Open access This is an open access article distributed in accordance with the Creative Commons Attribution Non Commercial (CC BY-NC 4.0) license, which permits others to distribute, remix, adapt, build upon this work non-commercially, and license their derivative works on different terms, provided the original work is properly cited, appropriate credit is given, any changes made indicated, and the use is non-commercial. See: http://creativecommons.org/licenses/by-nc/4.0/.

ORCID iD

Sadaf Kamil http://orcid.org/0000-0002-9370-8870

\section{REFERENCES}

1 Lau JF, Weinberg MD, Olin JW. Peripheral artery disease. Part 1: clinical evaluation and noninvasive diagnosis. Nat Rev Cardiol 2011:8:405-18.

2 Fowkes FGR, Rudan D, Rudan I, et al. Comparison of global estimates of prevalence and risk factors for peripheral artery disease in 2000 and 2010: a systematic review and analysis. Lancet 2013;382:1329-40.

3 O'Neal WT, Efird JT, Nazarian S, et al. Peripheral arterial disease and risk of atrial fibrillation and stroke: the multi-ethnic study of atherosclerosis. J Am Heart Assoc 2014;3:e001270.

4 Chang C-J, Chen Y-T, Liu C-S, et al. Atrial fibrillation increases the risk of peripheral arterial disease with relative complications and mortality: a population-based cohort study. Medicine 2016;95:e3002.

5 Selvin E, Erlinger TP. Prevalence of and risk factors for peripheral arterial disease in the United States: results from the National health and nutrition examination survey, 1999-2000. Circulation 2004;110:738-43.

6 Huang Y, Xu M, Xie L, et al. Obesity and peripheral arterial disease: a Mendelian randomization analysis. Atherosclerosis 2016;247:218-24.

7 Vrsalović M, Presečki AV. Atrial fibrillation and risk of cardiovascular events and mortality in patients with symptomatic peripheral artery disease: a meta-analysis of prospective studies. Clin Cardiol 2017;40:1231-5.

8 Thygesen LC, Daasnes C, Thaulow I, et al. Introduction to Danish (nationwide) registers on health and social issues: structure, access, legislation, and archiving. Scand J Public Health 2011;39:12-16.

9 Lynge E, Sandegaard JL, Rebolj M. The Danish national patient register. Scand J Public Health 2011;39:30-3.

10 Gaist D, Sørensen HT, Hallas J. The Danish prescription registries. Dan Med Bull 1997;44:445-8.

11 Lasota AN, Overvad K, Eriksen $\mathrm{HH}$, et al. Validity of peripheral arterial disease diagnoses in the Danish national patient registry. Eur $J$ Vasc Endovasc Surg 2017;53:679-85.

12 Olesen JB, Lip GYH, Hansen ML, et al. Validation of risk stratification schemes for predicting stroke and thromboembolism in patients with atrial fibrillation: nationwide cohort study. BMJ 2011;342:d124.

13 von Elm E, Altman DG, Egger M, et al. The strengthening the reporting of observational studies in epidemiology (STROBE) statement: guidelines for reporting observational studies. Int J Surg 2014;12:1495-9.

14 Malyar N, Freisinger E, Reinecke H. [Peripheral Arterial Disease - Trends in Morbidity and Mortality]. Dtsch Med Wochenschr 2018;143:766-70.

15 Hirsch AT, Haskal ZJ, Hertzer NR, et al. ACC/AHA 2005 practice guidelines for the management of patients with peripheral arterial disease (lower extremity, renal, mesenteric, and abdominal aortic): a collaborative report from the American association for vascular Surgery/Society for vascular surgery, Society for cardiovascular angiography and interventions, Society for vascular medicine and biology, society of interventional radiology, and the ACC/AHA Task force on practice guidelines (writing Committee to develop guidelines for the management of patients with peripheral arterial disease): endorsed by the American association of cardiovascular and pulmonary rehabilitation; National heart, lung, and blood Institute; Society for vascular nursing; transatlantic Inter-Society consensus; and vascular disease Foundation. Circulation 2006;113:e463-654.

16 Ciocan RA, Bolboacă SD, Rădulescu Şerban, et al. Demographic and comorbidity pattern of patients with critical limb ischemia. Folia Med 2017;59:14-22.

17 Shammas NW, Epidemiology SNW. Epidemiology, classification, and modifiable risk factors of peripheral arterial disease. Vasc Health Risk Manag 2007;3:229-34.

18 van Bussel BC, Schouten F, Henry RM, et al. Endothelial dysfunction and low-grade inflammation are associated with greater arterial stiffness over a 6-year period. Hypertension 2011;58:588-95.

19 Signorelli SS, Anzaldi M, Fiore V. Inflammation in peripheral arterial disease (pad). Curr Pharm Des 2012;18:4350-7.

20 Libby P, Ridker PM, Maseri A. Inflammation and atherosclerosis. Circulation 2002;105:1135-43. 
21 Cea-Soriano L, Fowkes FGR, Johansson S, et al. Time trends in peripheral artery disease incidence, prevalence and secondary preventive therapy: a cohort study in the health improvement network in the UK. BMJ Open 2018;8:e018184.

22 Willeit K, Kiechl S. Atherosclerosis and atrial fibrillation-two closely intertwined diseases. Atherosclerosis 2014;233:679-81.

23 Wasmer K, Unrath M, Köbe J, et al. Atrial fibrillation is a risk marker for worse in-hospital and long-term outcome in patients with peripheral artery disease. Int J Cardiol 2015;199:223-8.

24 Meves SH, Diehm C, Berger K, et al. Peripheral arterial disease as an independent predictor for excess stroke morbidity and mortality in primary-care patients: 5-year results of the getABI study. Cerebrovasc Dis 2010;29:546-54.

25 Patel NJ, Atti V, Mitrani RD, et al. Global rising trends of atrial fibrillation: a major public health concern. Heart 2018;104:1989-90.

26 Chugh SS, Havmoeller R, Narayanan K, et al. Worldwide epidemiology of atrial fibrillation: a global burden of disease 2010 study. Circulation 2014;129:837-47.

27 Demant MN, Andersson C, Ahlehoff O, et al. Temporal trends in stroke admissions in Denmark 1997-2009. BMC Neurol 2013;13:156.

28 Frost L, Vestergaard P, Mosekilde L, et al. Trends in incidence and mortality in the hospital diagnosis of atrial fibrillation or flutter in Denmark, 1980-1999. Int J Cardiol 2005;103:78-84.

29 Jespersen SF, Christensen LM, Christensen A, et al. Increasing rate of atrial fibrillation from 2003 to 2011 in patients with ischaemic stroke: results from 55,551 patients in a nationwide registry. Eur $J$ Neurol 2015;22:839-44.

30 Schmidt M, Ulrichsen SP, Pedersen L, et al. 30-Year nationwide trends in incidence of atrial fibrillation in Denmark and associated 5 -year risk of heart failure, stroke, and death. Int $J$ Cardiol 2016;225:30-6.
31 Schnabel RB, Yin X, Gona P, et al. 50 year trends in atrial fibrillation prevalence, incidence, risk factors, and mortality in the Framingham heart study: a cohort study. Lancet 2015;386:154-62.

32 Olinic D-M, Tataru DA, Homorodean C, et al. Antithrombotic treatment in peripheral artery disease. Vasa 2018;47:99-108.

33 Thiele I, Linseisen J, Heier M, et al. Time trends in stroke incidence and in prevalence of risk factors in southern Germany, 1989 to 2008/09. Sci Rep 2018;8:11981.

34 Anand SS, Bosch J, Eikelboom JW, et al. Rivaroxaban with or without aspirin in patients with stable peripheral or carotid artery disease: an international, randomised, double-blind, placebocontrolled trial. Lancet 2018;391:219-29.

35 Lackland DT, Roccella EJ, Deutsch AF, et al. Factors influencing the decline in stroke mortality: a statement from the American heart Association/American stroke association. Stroke 2014;45:315-53.

36 Chobanian AV, Bakris GL, Black HR, et al. Seventh report of the joint National Committee on prevention, detection, evaluation, and treatment of high blood pressure. Hypertension 2003;42:1206-52.

37 Itoga NK, Tawfik DS, Lee CK, et al. Association of blood pressure measurements with peripheral artery disease events. Circulation 2018;138:1805-14.

38 Kearney PM, Whelton M, Reynolds K, et al. Worldwide prevalence of hypertension: a systematic review. J Hypertens 2004;22:11-19.

39 Korhonen PE, Kautiainen H, Kantola I. Patients with resistant hypertension have more peripheral arterial disease than other uncontrolled hypertensives. J Hum Hypertens 2015;29:46-9.

40 Sundbøll J, Adelborg K, Mansfield KE, et al. Seventeen-Year nationwide trends in antihypertensive drug use in Denmark. Am J Cardiol 2017;120:2193-200. 